\title{
NEMO-Binding Domain Peptide ameliorates alveolar hypercoagulation and fibrinolytic inhibition in lipopolysaccharide-induced acute respiratory distress syndrome via NF-KB signaling pathway in mice
}

Yahui Wang

The People's Hospital of Weining County, Guizhou Province, China

\section{Yanqi Wu}

Guizhou Medical Unversity Affiliated Hospital

Bo Liu

Guizhou Medical Unversity Affiliated Hospital

Huilin Yang

Guizhou Medical University

Hong Qian

Guizhou Medical University

\section{Yumei Cheng}

Guizhou Medical University Affiliated Hospital

\section{Xiang Li}

Guizhou Medical University

\section{Guixia Yang}

Guizhou Medical University

\section{Xinghao Zheng}

Guizhou Medical University

Feng Shen ( $\nabla$ doctorshenfeng@163.com )

Guizhou Medical University Affiliated Hospital https://orcid.org/0000-0002-0807-8617

\section{Research}

Keywords: Acute respiratory distress syndrome, NEMO-Binding Domain Peptide, NF-KB signal pathway, Alveolar hypercoagulation, Fibrinolytic inhibition

Posted Date: December 14th, 2020

DOI: https://doi.org/10.21203/rs.3.rs-38826/v2 
License: (c) (i) This work is licensed under a Creative Commons Attribution 4.0 International License. Read Full License 


\section{Abstract}

Background It was confirmed that alveolar hypercoagulation and fibrinolytic inhibition were associated with refractory hypoxemia in acute respiratory distress syndrome (ARDS), and NF-kB pathway was involved in this process. The purpose of the present study is to explore the effects and relevant mechanisms exerted by NEMO-binding domain peptide (NBDP) to alleviate alveolar hypercoagulation and fibrinolytic inhibition aroused by lipopolysaccharide in ARDS mice. Materials and Methods Adult male BALB/c mice inhaled lipopolysaccharide (LPS, mg/L) to induce ARDS. 30 minutes before LPS administration, we treated the mice with increasing concentrations of intratracheally inhaled NBDP or saline aerosol. Six hours after LPS treatment, bronchoalveolar lavage fluids (BALF) were collected and then all mice were executed. We checked coagulation and fibrinolysis associated factors in lung tissues and in BALF as well. We simultaneously observed the activation of NF-KB signaling pathway as well. Results NBDP pretreatment dose-dependently inhibited either the expressions of tissue factor (TF) and plasminogen activator inhibitor (PAI) 1 in lung tissues or the secretions of TF, PAI-1, thrombinantithrombin complex (TAT) and promoted activated protein C (APC) secretion in BALF induced by LPS. LPS-induced high expression of pulmonary procollagen peptide type $₫(P 区 P)$ was also declined by NBDP pretreatment in dose-dependent manner. Western blotting showed that NBDP pretreatment obviously attenuated LPS-induced IKKa/ $\beta$, IKa and NF-KB p65 activation. LPS-induced p65 DNA binding activity was inhibited by NBDP pretreatment either. We also noticed NBDP protected mice against LPS-induced lung injury in a dose-dependent manner. Conclusions Our experimental findings demonstrate that NBDP dose-dependently ameliorated LPS-induced alveolar hypercoagulation and fibrinolytic inhibition through inhibiting NF-KB signaling pathway. NBDP is expected to be a new therapeutic target to correct the abnormalities of alveolar coagulation and fibrinolysis in ARDS.

\section{Background}

Acute lung injury (ALI) and its severe form, respiratory distress syndrome (ARDS) is one of the most common diseases admitted to the intensive care unit (ICU) and is the leading cause of respiratory failure and death in critically ill patients as well [1]. One of the important reasons keeping the treatment of ARDS difficult and the mortality rate high is the complicated pathophysiology, among which alveolar hypercoagulation and fibrinolytic inhibition are crucial [2]. Alveolar hypercoagulation and fibrinolysis inhibition resulted in extensive microthrombus formation in pulmonary vessels, large amount of fibrin deposition in airspace[3-4], which were closely related with decrease of lung compliance, V/Q ratio imbalance and arteriovenous shunt, resulting in refractory hypoxemia in ARDS. But so far, no satisfatory drug is available for managing the hypercoagulation and fibrinolytic inhibition in ARDS because of the complicated mechanisms underlying these pathological process. From our previous studies $[5,6]$ and other published data [7], it is accepted that NF-KB signaling pathway plays a pivotal role in pathogenesis of alveolar hypercoagulation and fibrinolytic inhibition.

Nuclear factor kappa B (NF-kB) participates substantially in the regulation of many important physiopathologic processes, immune, inflammatory, tumorigenesis and stress response[8, 9]. NF-KB 
activation results in its translocation from the cytoplasm to the nucleus. Under normal conditions, NF-KB is sequestered in the cytoplasm, bound by members of its inhibitor proteins, which comprises ІкBa, ІкB $\beta$ and IKBY. IKB kinase (IKK) complex, necessary for NF-KB activation, comprises three subunits, IKKa, IKK $\beta$ and IKKY, of which IKKY is also known as NEMO. NEMO does not have catalytic domain itself, but plays a pivotal role in biology as being a part of the IKK complex[10,11]. The NH2-terminus of NEMO associates with a hexapeptide sequence, Leu-Asp-Trp-Ser-Trp-Leu, within the $\mathrm{COOH}$ terminus of IKKa and IKK $\beta$ called the NEMO-binding domain (NBD) [12], which is the basic structure for the crosstalk among IKKa, IKK $\beta$ and NEMO, maintaining the biological activity of IKK complex [13]. Though many methods could be used to inhibit NF-KB, such as NF-KB /IKK $\beta$ gene knockout or knockdown, specific inhibitor of NF-kB, blocking p65 translocation from cytoplasm into nucleus or blocking the binding of p65 to its specific DNA sequence ( $\mathrm{KB}$ sequence) etc., some basal biological activities of NF-KB would also inevitably be inhibited by these methods $[14,15]$. A small molecular NBD peptide (NBDP), however, was confirmed not only to selectively inhibit the NF-KB-mediated target gene transcription through targeting the crosstalk of IKK-NEMO[16], but also to maintain the important basal activities of NF-KB [17]. Previous studies have demonstrated that NBDP effectively inhibited NF-KB pathway activation[18,19,20,21], indicating NBDP might be efficacious in correcting alveolar coagulation and fibrinolysis abnormalities via NF-KB pathway in ARDS. In our study, it demonstrated that NBDP dose-dependently attenuated LPS-induced alveolar hypercoagulation and fibrinolytic inhibition by inactivating NF-KB signaling pathway in mice, and NBDP is expected to be a new target in the treatment of ARDS.

\section{Materials And Methods Experimental animal}

BALB/c male mice, aged 6-8 weeks and weighing 20-25 g, were purchased from the Animal Center of Guizhou Medical University. All mice were fed a normal standard diet at a controlled environment (temperature $22 \pm 1^{\circ} \mathrm{C}$ ) with 12 hours light/dark cycles and controlled humidity. The mice were given 7 days to acclimatize to the environment prior to the experiment. The study was approved by the Animal Ethics Committee of Guizhou Medical University and conducted in accordance with provisions of the Chinese Laboratory Animal Management Regulations.

\section{Animal model}

ARDS was made by LPS aerosol inhalation. 72 mice were randomly divided into six groups: Control, Model, N-NBD, L-NBD, M-NBD and H-NBD, with 12 in each group. The mice in Model, N-NBD, L-NBD, MNBD and H-NBD received $50 \mu \mathrm{l}$ of LPS (4 $\mathrm{mg} / \mathrm{ml}$, Sigma-Aldrich) while mice in control group received same volume of saline inhalation. 30 minutes before LPS administration, the mice in L-NBD, M-NBD and H-NBD group inhaled $50 \mu \mathrm{l}$ of NBDP (MERCK) with concentration of $120 \mu \mathrm{g} / \mathrm{ml}, 240 \mu \mathrm{g} / \mathrm{ml}$ and $360 \mu \mathrm{g} / \mathrm{ml}$, respectively. N-NBD group served as negative control and received a non-functional NBDP analogue ( $50 \mu \mathrm{l}$, MERCK) at concentration of $240 \mu \mathrm{g} / \mathrm{ml} .6$ hours after LPS or saline inhalation, all mice were euthanized under anesthetization with chloral hydrate. Bronchoalveolar lavage fluid (BALF) samples 
were collected for coagulation-related factor detection. Left lung tissues were collected for histopathological and immunohistochemical analysis while right lungs were rapidly frozen in liquid nitrogen and stored at $-80^{\circ} \mathrm{C}$ for enzyme-linked immunosorbent assay (ELISA) and western blot(WB) analysis.

\section{Real-time quantitative PCR}

Real-time quantitative PCR (qPCR) was performed to detect TF and PAl-1 gene expression. The total RNA concentration was evaluated by using a NanoDrop-2000 spectrophotometer (NanoDrop Technologies, Germany) and the A260/A280 ratio of extracted RNA was controlled between 1.8 to 2.0. Primers were designed based on the TF and PAI-1 gene sequences supplied by NCBI gene database. The primers were synthesized by Guangzhou Aiji Biotechnology Co., Ltd (Table 1). We performed PCR amplification using CDNA as template. The reaction system was set as follows: SYBR Green Mix $10 \mu$, forward primer and reverse primer $0.8 \mu \mathrm{l}$ respectively, cDNA template $0.8 \mu \mathrm{l}$, ddH2O $7.6 \mu \mathrm{l}$, which were synthesized into a system containing $20 \mu \mathrm{l}$ reagents. Dissolution and amplification curve of the gene were recorded following the gene amplification. Expressions of target genes were calculated using the $2^{-} \Delta \Delta \mathrm{Ct}$ method.

Table 1

Gene sequences of TF, PAl-1

\begin{tabular}{|ll|}
\hline Gene & Sequences \\
\hline TF & 5-AGA CGG AGA CCA ACT TGT GAT-3 \\
& 5-CTG CTG AAT TAC TGG CTG TCC-3 \\
PAI-1 & 5-CTG CAA AAG GTC AGG ATC GAG-3 \\
& 5-CAT CAC TTG GCC CAT GAA GAG-3 \\
\hline B-actin & 5-CAC CCG CGA GTA CAA CCT TC-3 \\
& 5-CCA ATA CCC ACC ATC ACA CC-3 \\
\hline Lengends for figures \\
\hline
\end{tabular}

\section{Western blotting (WB)}

Cytoplasmic proteins were extracted using the Cell Solute Extraction Kit according to the manufacturer's instructions (Solebao Technology Co., Ltd, Beijing, China). Briefly, the concentration of protein was measured using a BCA assay kit. An equal amount of protein from each sample was resolved in $12 \%$ Trisglycine SDS polyacrylamide gel. The protein band was blotted onto a nitrocellulose membrane. After incubating for 2 hours in blocking solution, the membrane was incubated with $p$-p65, $p$-IKKa/ $\beta$, p-IKB (Cell Signaling Technology), PAI-1, TF and PIIIP (Abcam) antibodies (1:1000 dilution) for 24 hours. Then a secondary antibody (1:200dilution) (horseradish peroxidase-conjugated goat anti-rabbit immunoglobulin) was added and incubated for 2 hours at $37^{\circ} \mathrm{C}$. The target protein bands were visualized using an 
enhanced chemiluminescence detection system (Millipore, MA, USA). Relative band densities were quantified by Image $\mathrm{J}$ software.

\section{ELISA assay}

The BALF was centrifuged at $4500 \mathrm{rpm}$ for 10 minutes at $4{ }^{\circ} \mathrm{C}$, then the supernatant was collected and stored at $-80^{\circ} \mathrm{C}$ for test. Tissue factor (TF), plasminogen activator inhibitor-1(PAl-1) and activated protein C(APC) levels were determined by using ELISA kits (Huamei Bio-company, Wuhan, China) according to the manufacturer's instructions.

\section{Histopathology}

The lung tissues were fixed with $4 \%$ paraformaldehyde for 24 hours, then dehydrated, embedded in paraffin and sliced $(4 \mu \mathrm{m})$. Then the slices were stained with hematoxylin-eosin (H\&E). The scores of lung injury were blindly evaluated by pathologists, as described previously [22]. Each histological change was scored (lung injury score, LIS) from 0 to 3 according to the lesion range, including alveolar wall thickening, edema, inflammatory cell infiltration, hemorrhage and cellulose deposition (0: normal, 1: injury $\leq 25 \%$ of the field, 2 : injury in $25 \%-50 \%$ of the field, 3 : injury $\geq 50 \%$ of the field).

\section{NF-KB p65 DNA binding activity}

The right upper lung was used for NF-KB p65 DNA binding activity test according to the manufacturer's instructions for the Universal EZ-TFA Transcription Factor Chemiluminescence Kit. The nuclear extract of lung tissues was added to a plate containing biotinylated oligonucleotide which had NF-KB binding site. After incubating for 1 hour at room temperature, the plates were washed and incubated with rabbit antiNF-KB p65 (1:1000 dilution) for 1 hour. After washing the plates, anti-rabbit horseradish peroxidaseconjugated antibody (1:500 dilution) was added and incubated for 30 minutes. Then adding the chemiluminescent substrate solution and incubated for 5 minutes. The sample OD value was read in a microplate reader at 1 -s integration time.

\section{Immunohistochemistry (IHC)}

The paraffin sections were rehydrated using a series of ethanol, and the antigen was retrieved using citrate buffer. The nonspecific binding site was blocked by $3 \%$ BSA. The sections were incubated with rabbit anti-mouse p65 (1:600 dilution) and type III collagen (1:200 dilution) antibody at $4^{\circ} \mathrm{C}$ overnight, followed by washing and incubating with corresponding HRP-labeled secondary antibodies for 1 hour at room temperature. The antigen expressions were visualized using peroxidase activity developed by DAB staining solution and observed at a magnification of 400 .

\section{Statistical analysis}

Statistical analyses were performed with SPSS. Data was expressed as mean \pm SD. Statistical differences were determined by one-way analysis of variance (ANOVA) and the Student-Newman-Keuls (SNK) method. $\mathrm{P}<0.05$ was considered to be statistically significant. 


\section{Results}

NBDP improved pulmonary pathological changes induced by LPS inhalation in mice To assess pulmonary pathological changes and the degree of lung injury, HE staining and histopathological analysis were performed. LPS provoked excessive edema, obvious inflammatory cell infiltration, alveolar collapse, alveolar wall thickness and severe hemorrhage, which were all dosedependently inhibited by NBDP (Fig. 1). The LPS-induced high W/D ratio and the high LIS also significantly decreased with NBDP treatment (Fig. 2).

NBDP attenuated expressions of TF and PAI-1 in mRNA and in protein levels in LPS-induced ARDS mice In order to evaluate the coagulation and fibrinolytic status in LPS-induced lung tissues, TF and PAl-1 were measured by quantitative PCR and WB. Data showed that LPS stimulated high expressions of TF and PAl-1 both in mRNA and in protein in pulmonary tissues. NBDP pretreatment effectively attenuated TF and PAl-1 expressions arisen by LPS (Fig. 3).

NBDP inhibited the secretions of TF, PAI-1, TAT and promoted APC production from LPS-induced lung tissue

Concentrations of TF, PAI-1, TAT and APC in BALF were determined by ELISA, so as to evaluate the alveolar hypercoagulation and fibrinolytic inhibition. LPS stimulation for 6 hours resulted in obvious increases in TF, PAI-1 and TAT levels and a decrease of APC level in BALF, which were all reversed by NBDP in dose-dependent manner (Fig. 4).

NBDP alleviated PIIIP deposition in lung tissues arisen by LPS induction in mice In order to evaluate the fibrinolystic inhibition in pulmonary tissues in condition of LPS treatment, PIIIP level in lung tissues was checked by using immunohisto-

chemistry. Results demonstrated that LPS induced a large amount of PIIIP in pulmonary tissues. After pretreatment with NBD, LPS-induced PIIIP deposition was significantly reduced in a dose-dependent manner. (Fig. 5)

NBDP inhibited NF-kB activation induced by LPS

To assess the effects of NBDP on NF-KB signaling pathway, we performed western blotting to detect the phosphorylation of IKKa/ $\beta$, IKBa and p65 after LPS stimulation. The phosphorylation levels of IKKa/ $\beta$ (p$\mathrm{IKKa} / \beta), \mathrm{p}-\mathrm{IKBa}$ and $\mathrm{p}-\mathrm{p} 65$ increased significantly in LPS-induced lung tissues. However, these increases were weakened by NBDP pretreatment. (Fig. 6)

NBDP decreased p65 DNA binding activity initiated by LPS stimulation NF-KB p65 DNA binding activity stands for p65 translocation from cytoplasm to nucleus. Our data showed that NF-KB p65 DNA binding activity was significantly increased after LPS stimulation, which was dose-dependently abolished by NBDP. (Fig. 7) 


\section{Discussion}

Results of our study demonstrated the efficacies of NBDP on LPS-induced alveolar hypercoagulation and fibrinolysis inhibition in mice. We found that NBDP effectively ameliorated LPS-induced hypercoagulation and fibrinolysis inhibition in lung tissues and in airspace as well. We also observed that NBDP pretreatment attenuated LPS-induced lung injury, indicated by improvements in pathological changes, W/D ratio and in LIS, which was consistent with the results finished by Huang et al [20]. Finally, we noticed that NBDP effectively inhibited NF-KB pathway activation induced by LPS.

In this experiment, LPS aerosol inhalation promoted obvious pulmonary edema, alveolar collapes, pulmonary hemorrhage in mice, which mimicked the pathogenesis of ARDS [23], indicating ARDS model being successfully set up. The result was consistent with our previous study [22].

TF is a potent procoagulant that initiates the extrinsic coagulation cascade mainly through interacting

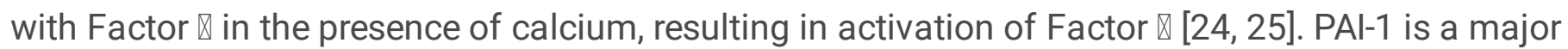
physiological inhibitor of the fibrinolytic system, which also regulates thrombosis[26]. PAl-1 binds to and inhibits tissue and urokinase-type plasminogen activators (tPA and UPA), thereby reducing plasmin production and fibrin clot lysis [27]. The results of our study showed that both TF and PAI-1, either in mRNA or in protein, highly expressed in pulmonary tissue under LPS stimulation, indicating a procoagulation and fibrinolytic defect in lung tissue [28].

Thrombin-antithrombin (TAT) is a complex of thrombin and antithrombin that directly reflects thrombin generation. An increase in TAT suggests a state of procoagulant activity [29]. APC is a protein synthesized by the liver and exerts anticoagulant activity by hydrolyzing blood coagulation factors $\mathrm{Va}$ and VIIIa [30]. Our experimental data showed that in BALF, the concentrations of TF, PAI-1, TAT all significantly elevated, while APC concentration statistically decreased, implying the hypercoagulation and fibrinolytic inhibition in airspace being in LPS-stimulated ARDS [31].

PIIIP is mainly synthesized and secreted by fibroblasts and transformed myofibroblasts. In addition, PIIIP is the main component of extracellular matrix (ECM), and the excessive accumulation of PIIIP stands for pulmonary fibrous deposition. High expression of pulmonary PIIIP under LPS treatment in our study signified an increased fibrous tissues in lung because of fibrinolytic inhibition.

NBDP is a protein peptide that has been shown to inhibit the activation of the classical NF-KB signaling pathway by interfering NEMO-IKKa/IKK interaction [32]. Our data demonstrated that pretreatment with NBDP markedly inhibited NF-KB pathway activation induced by LPS provocation, shown by decreased levels of $p-I K K a / \beta, p$-IKa and p-p65, and by the decreased p 65 NDA binding activity as well. At same time, NBDP also effectively suppressed TF and PAl-1 expressions in pulmonary tissue, as well as secretions of TF, PAI-1 and TAT in BALF while promoted APC production in BALF. Therefore, we have the reason to think that NBDP corrected alveolar hypercoagulation and fibrinolytic inhibition induced by LPS via NF-KB pathway inactivation. Interestingly, we found that the higher the dose of NBDP, the more obvious the 
efficacies of NBDP on coagulation and fibrinolysis associated factors and on NF-KB inactivation as well, indicating a dose-dependent manner.

Unlike other inhibitors or methods, such as gene knockdown, knockout or specific inhibitor, it has been testified that NBDP selectively inhibit NF-KB-mediated target gene transcription while the important basal acitvities of NF-KB being maintained $[16,17]$, which made NBDP much more attractive to be a new potential therapeutic targat in ARDS treatment.

In our experiment, we set a negative control group with non-functional NBDP analogue (50 $\mu$, MERCK), such that we eliminated the affection of NBDP itself on the results of our study.

\section{Conclusions}

NBDP dose-dependently ameliorated alveolar hypercoagulation and fibrinolysis inhibition via NF-KB signaling pathway. NBDP is expected a new effective therapeutic target in ARDS.

\section{Abbreviation}

NBDP, NEMO-Binding Domain Peptide; ARDS, acute respiratory distress syndrome; ALI, acute lung injury; $B A L F$, bronchoalveolar lavage fluids; TF, tissue factor; PAl-1, plasminogen activator inhibitor 1; TAT,

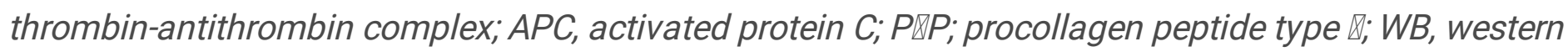
blotting; IHC, immunohistochemistry; tPA, tissue plasmiogen activator; uPA, urokinase-type plasminogen activators.

\section{Declarations}

\section{Ethics approval and consent to participate}

The whole experiment performed in this study was conformed to the Guide for the Care and Use of Laboratory Animals and were approved by the Institutional Animal Care and Use Committee.

\section{Consent for publication}

All authors consent to publicate the manuscript in Respiratory Research.

\section{Availability of data and material}

We could offer the data and material if there is any requirement.

\section{Funding}

This study was supported by the grants from Guizhou Science and Technology Plan Project ([2019]1261); Natural Science Foundation of Guizhou Province [2017]7217. 


\section{Competing interests}

The authors declare that they have no competing interests.

\section{Author Contributions}

Yahui Wang and Yanqi WU performed the whole the experimental operation, finished the statistical analysis, and Yanqi WU wrote the primary manuscript. Bo LIU, Huilin YANG, Hong QIAN, Yumei CHENG, Xiang LI, Guixia YANG, Xinghao were mainly responsible for the data collection. Feng Shen conceived the whole design of the study, analyzed the data and organized the final manuscript. All authors read and approved the final manuscript.

\section{Acknowledgements}

We give much thanks to Mr. Yi FANG and Professor Xu LIU, who give much help to our experiment.

\section{References}

1. Bellani G, Laffey J G, Tài Pham, et al. Epidemiology, patterns of care, and mortality for patients with acute respiratory distress syndrome in intensive care units in 50 countries[J]. The Journal of the American Medical Association, 2016, 315(8):788-800.

2. Nekari de LC, Isabel SC, Uriarte UXB, Maria PRC, Celorrio LA. Acute respiratory distress syndrome: a review of the Berlin definition[J]. Revista Espanola De Anestesiologia Y Reanimacion, 2014, 61(6):319.

3. Mokra D, Kosutova P. Biomarkers in acute lung injury[J]. Respiratory Physiology \& Neurobiology, 2015, 209(4):52-58.

4. Ware L B, Matthay M A. The acute respiratory distress syndrome[J]. New England Journal of Medicine, 2000, 342(18):1334.

5. Liu B, Wu Y, Wang Y, et al. NF-kB p65 Knock-down inhibits TF, PAl-1 and promotes activated protein C production in lipopolysaccharide-stimulated alveolar epithelial cells type II [J]. Exp lung Res. 2018,19:1-11.

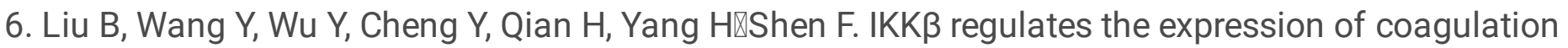
and fibrinolysis factors through the NF-KB canonical pathway in LPS-stimulated alveolar epithelial cells type II. Experimental and Therapeutic Medicine, 2019, 18(4): 2859-2866.

7. Ding R, Zhao D, Li X, Liu B, Ma X. Rho-kinase inhibitor treatment prevents pulmonary inflammation and coagulation in lipopolysaccharide-induced lung inury. Thrombosis Research, 2017, 150:59-64.

8. Bonizzi G, Karin M. The two NF-kB activation pathways and their role in innate and adaptive immunity[J]. Trends Immunol, 2004, 25:280-288.

9. Hayden MS, Ghosh S. Signaling to NF-KB[J]. Genes Dev, 2004, 18: 2195-2224.

10. Zheng C, Yin Q, Wu H. Structural studies of NF-kB signaling. Cell Research, 2011, 21:183-195. 
11. Israel A. The IKK complex, a central regulator of NF-KB activation. Cold Spring Harb Perspect Biol, 2010, 2:a000158.

12. May M J, Marienfeld R B, Ghosh S. Characterization of the IKB-kinase NEMO Binding Domain[J]. Journal of Biological Chemistry, 2002, 277(48):45992-46000.

13. Kensche T, Tokunaga F, Ikeda F, Goto E, Iwai K, Dikic I. Analysis of nuclear factor-KB (NF-KB) essential modulator (NEMO) binding to linear and lysine-linked ubiquitin chains and its role in the activation of NF-kB. The Journal of Biological Chemistry, 2012, 287(28): 23626-23634.

14. Ward C, Schlingmann B, Stecenko AA, Guidot DM, Koval M. NF-kB inhibitors impair lung epithelial tight junctions in the absence of inflammation. Tissue Barriers, 2015, 3:1-2, e982424.

15. Zhang Q, Lenardo MJ, Baltimore D. 30 years of NF-kB: a blossoming of relevance to human pathobiology. Cell, 2017, 168(1-2): 37-57.

16. May, MJ. Selective inhibition of NF-kappa B activation by a peptide that blocks the interaction of NEMO with the lkappa B kinase complex. ence, 2000, 289(5484):1550-1554.

17. Khaja K, Robbins P. Comparison of functional protein transduction domains using the NEMO binding domain peptide. Pharmaceuticals, 2010, 3: 110-124.

18. O'Donnell MA, Hase H, Legarda D, Ting AT. NEMO inhibits programmed necrosis in an NFKBindependent manner by restraining RIP1. PloS ONE, 2012, 7(7): e41238.doi:10.1371/joumal. pone.0041238.

19. Zilberman-Rudenko J, Shawver LM, Wessel AW, Luo Y, Pelletier M, Tsai WL, Lee Y, Vonortas S, Cheng $\mathrm{L}$, Ashwell JD, et al. Recruitment of A20 by the C-terminal domain of NEMO suppresses NF-KB activation and autoinflammatory disease. PNAS, 2016, 113(6): 1612-1617.

20. Huang J, Li L, Weifeng Y, et al. NEMO-binding domain peptide attenuates lipopolysaccharideinduced acute lung injury by inhibiting the NF-KB signaling pathway[J]. Mediators of Inflammation, 2016, 2016:1-11.

21. Zhuang Z, Li H, Lee H, Aguilar M, Gocho T, Ju H, Lida T, Ling J, Fu J, Wu m, Sun Y, Lu Y, Chiao PJ. NEMO peptide inhibitts the growth of pancreatic ductal adenocarcinoma by blocking NF-KB activation. Cancer Lett, 2017, 411:44-56.

22. Wu Y, Wang Y, Liu B, Cheng Y, Qi H, Yang H, Li X, Yang G, Zheng X, Shen F. SN50 attenuates alveolar hypercoagulation and fibrinolysis inhibition in acute respiratory distress syndrome mice through inhibiting NF-KB p65 translocation. Respiratory Research, 2020,21:130.

23. Schuster D P. ARDS: clinical lessons from the oleic acid model of acute lung injury. American Journal of Respiratory and Critical Care Medicine, 1994, 149, (1): 245-260.

24. Bastarache JA, Wang L, Geiser T, Wang Z, Albertine KH, Matthay MA, Ware LB. The alveolar apithelium can initiate the extrinsic coagulation cascade through expression of tissue factor. Thorax, 2007, 62:608-616.

25. Ahmad S, Ahmad A, Rancourt RC, Neeves KB, Loader JE, Hendry-Hofer T, Paola JD, Reynolds SD, White CW. Tissue factor signals airway epithelial basal cell survival via coagulation and proteaseactivated receptor. Am J Respir Cell Mol Biol, 2013, 48(1): 94-104. 
26. Poole L G, Massey V L, Siow D L, et al. Plasminogen Activator Inhibitor-1 is Critical in Alcoholenhanced Acute Lung Injury in Mice[J]. American Journal of Respiratory Cell and Molecular Biology, 2017, 57(3):315-323.

27. Damare J, Brandal S, Fortenberry Y M. Inhibition of PAl-1 Antiproteolytic Activity Against tPA by RNA Aptamers[J]. Nucleic Acid Therapeutics, 2014, 24(4):239-249.

28. Idell S. Coagulation, fibrinolysis, and fibrin deposition in acute lung injury. Crit Care Med, 2003, 31[Suppl.]: S213-S220.

29. Lee $S$ Y, Niikura T, Iwakura T, et al. Thrombin-antithrombin III complex tests[J]. Journal of Orthopaedic Surgery, 2017, 25(1):1-6.

30. Christiaans SC, Wagener BM, Esmon CT, et al. Protein C and acute inflammation: a clinical and biological perspective[J]. Am J Physiol Lung Cell Mol Physiol, 2013, 305(7):L455-466.

31. Camprubi-Rimblas M, Tantinya N, Bringue J, Guillamat-Prats R, Artigas A. Anticoagulant therapy in acute respiratory distress syndrome. Ann Transl Med, 2018, 6(2): 36.

32. Dai S, Hirayama T, Abbas S, et al. The IKB kinase (IKK) inhibitor, NEMO-binding domain peptide, blocks osteoclastogenesis and bone erosion in inflammatory arthritis[J]. Journal of Biological Chemistry, 2004, 279(36):37219-37222.

\section{Figures}
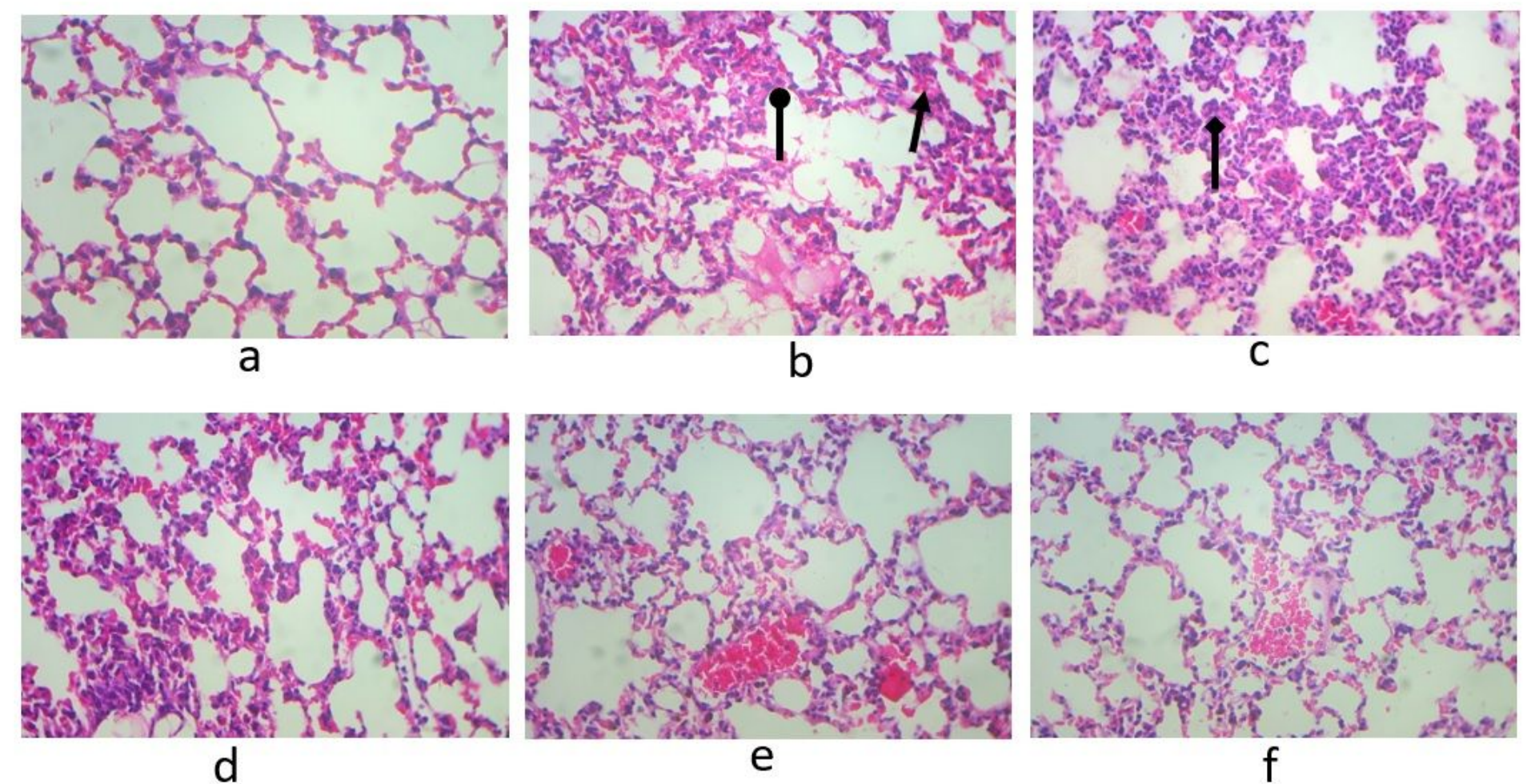

\section{Figure 1}


Pathological changes in the lung tissues $(\times 100)$. Lung sections were stained with hematoxylin and eosin 6 hours after LPS administration. LPS destroyed the lung tissues, resulting in hemorrhage ( ), inflammatory cell infiltration ( ), alveolar wall thickening ( ) and so on, which were all attenuated by NBDP pretreatment (a: Control, b: Model, c: N-NBD, d: L-NBD, e: M-NBD, f: H-NBD)
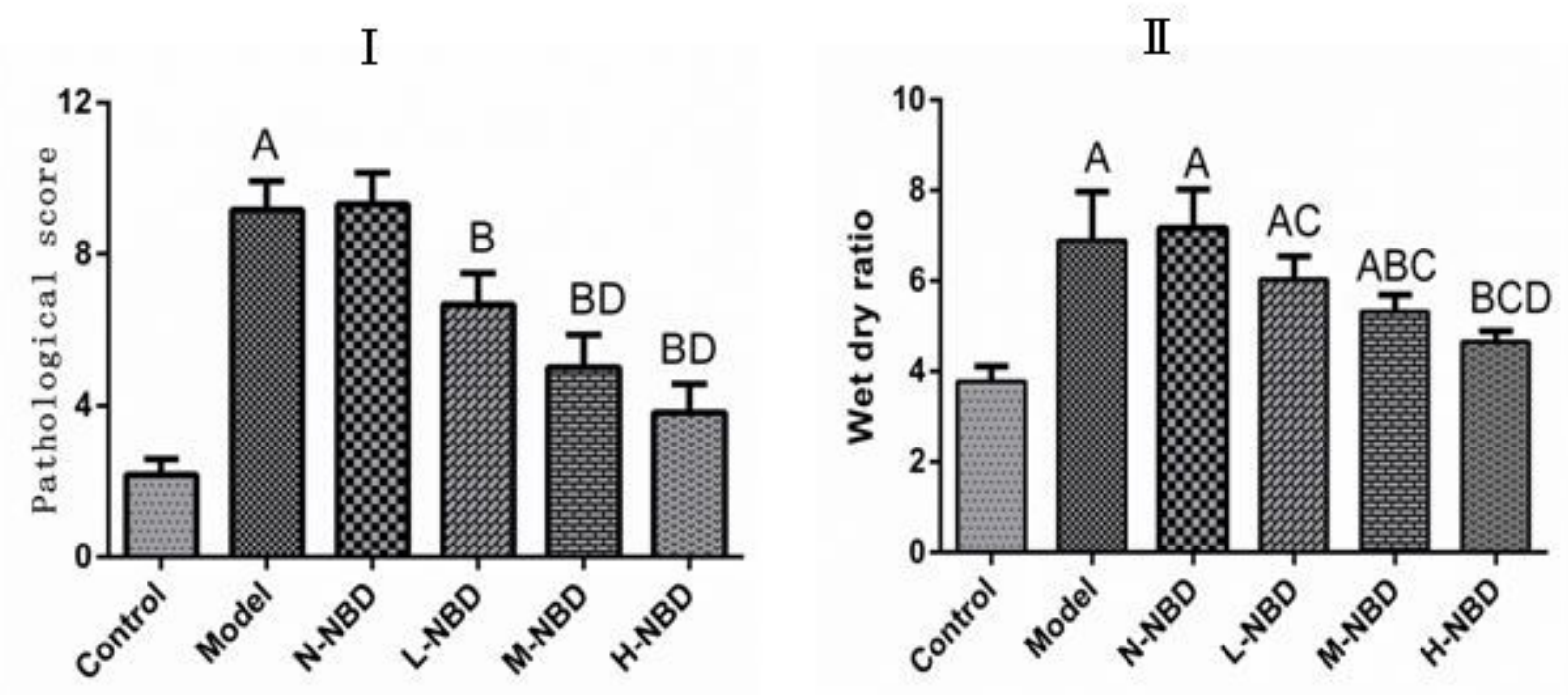

Figure 2

NBDP alleviated lung injury caused by LPS. LPS inhalation resulted in obvious pathological changes and severe pulmonary edema. NBDP pretreatment significantly alleviated these pathological changes (Figure

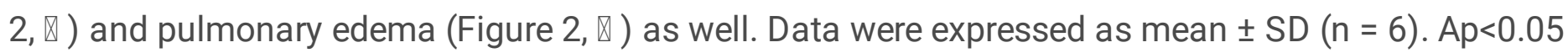
compared with Control. Bp $<0.05$ compared with Model. $\mathrm{Cp}<0.05$ compared with N-NBD. $\mathrm{Dp}<0.05$ compared with L-NBD. 


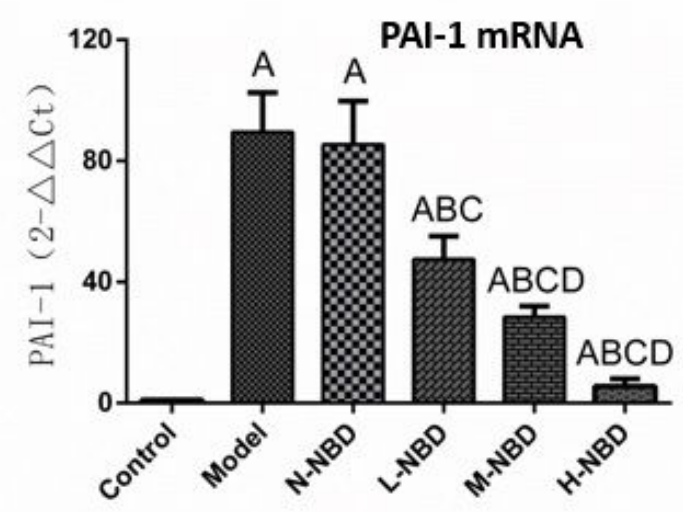

PAI-1 protein

Control Model N-NBD L-NBD M-NBD H-NBD
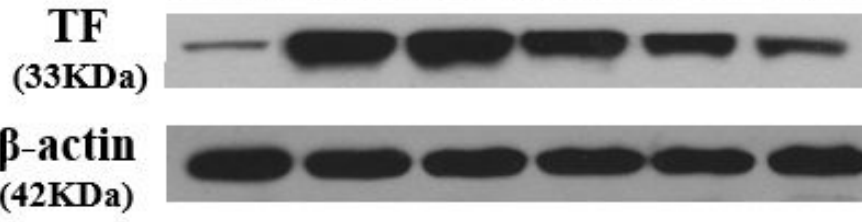

\section{(42KDa)}
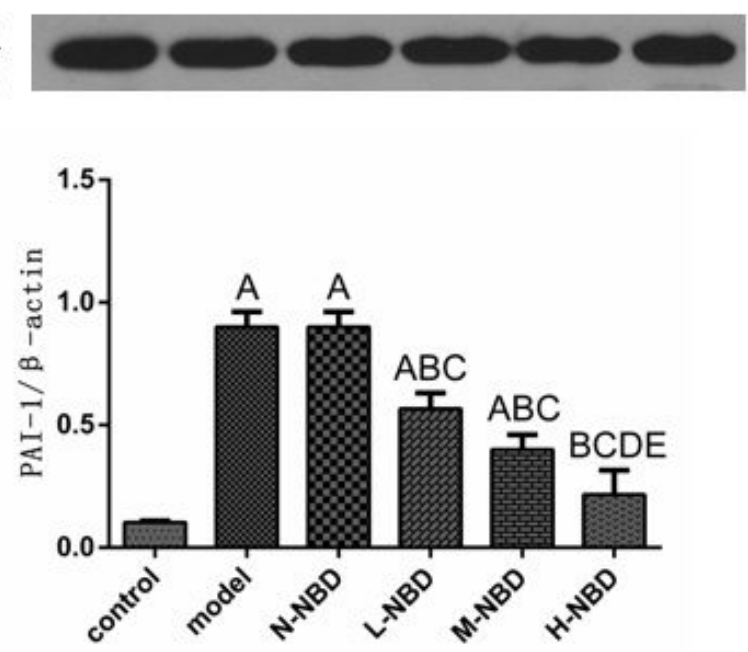

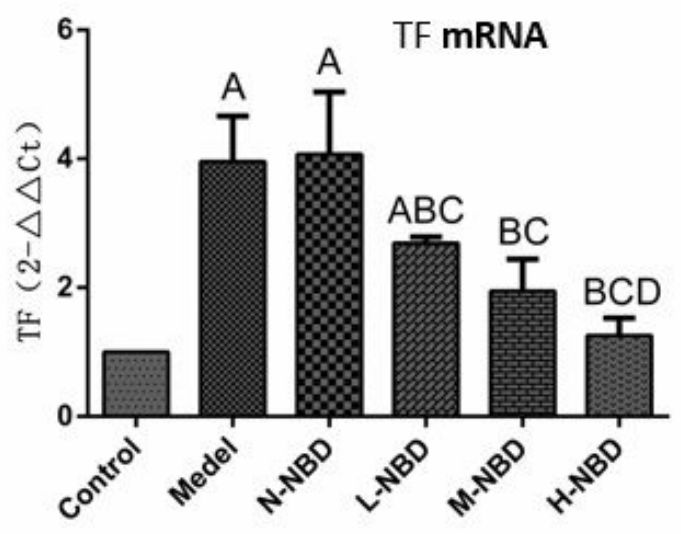

TF protein

Control Model N-NBD L-NBD M-NBD H-NBD

PAI-1

(45KDa)

$\beta$-actin

(42KDa)

\section{Figure 3}

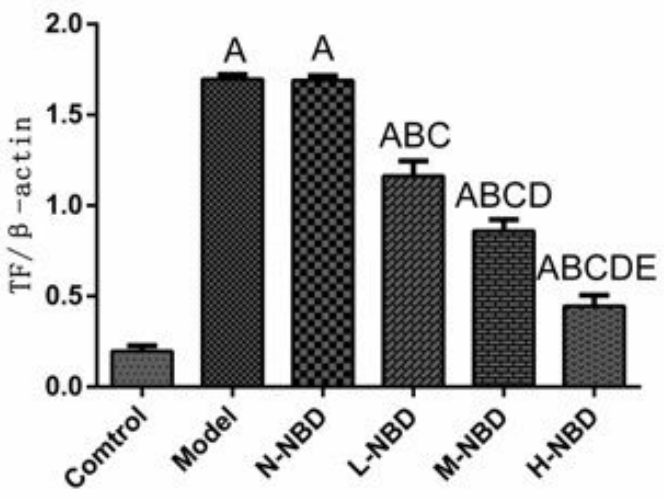

Changes of TF and PAl-1 expressions in pulmonary tissue. 6 hours after LPS inhalation with or without NBDP pretreatment, mice were exsanguinated and the lungs were collected. RT-PCR was performed to detect TF and PAl-1 mRNA expression in lung tissues. TF and PAI-1 mRNA level was expressed as a ratio of TF or PAl-1 grey value to $\beta$-actin. Western blotting was performed to detect TF and PAl-1 protein expressions in lung tissues. TF and PAI-1 protein levels was calculated as a ratio of intensities of TF or PAl- 1 to the corresponding $\beta$-actin bands. Each bar represents the mean \pm SD of 6 mice. Ap $<0.05$ compared with Control. Bp<0.05 compared with Model. Cp $<0.05$ compared with N-NBD. Dp $<0.05$ compared with L-NBD. Ep<0.05 compared with M-NBD. 

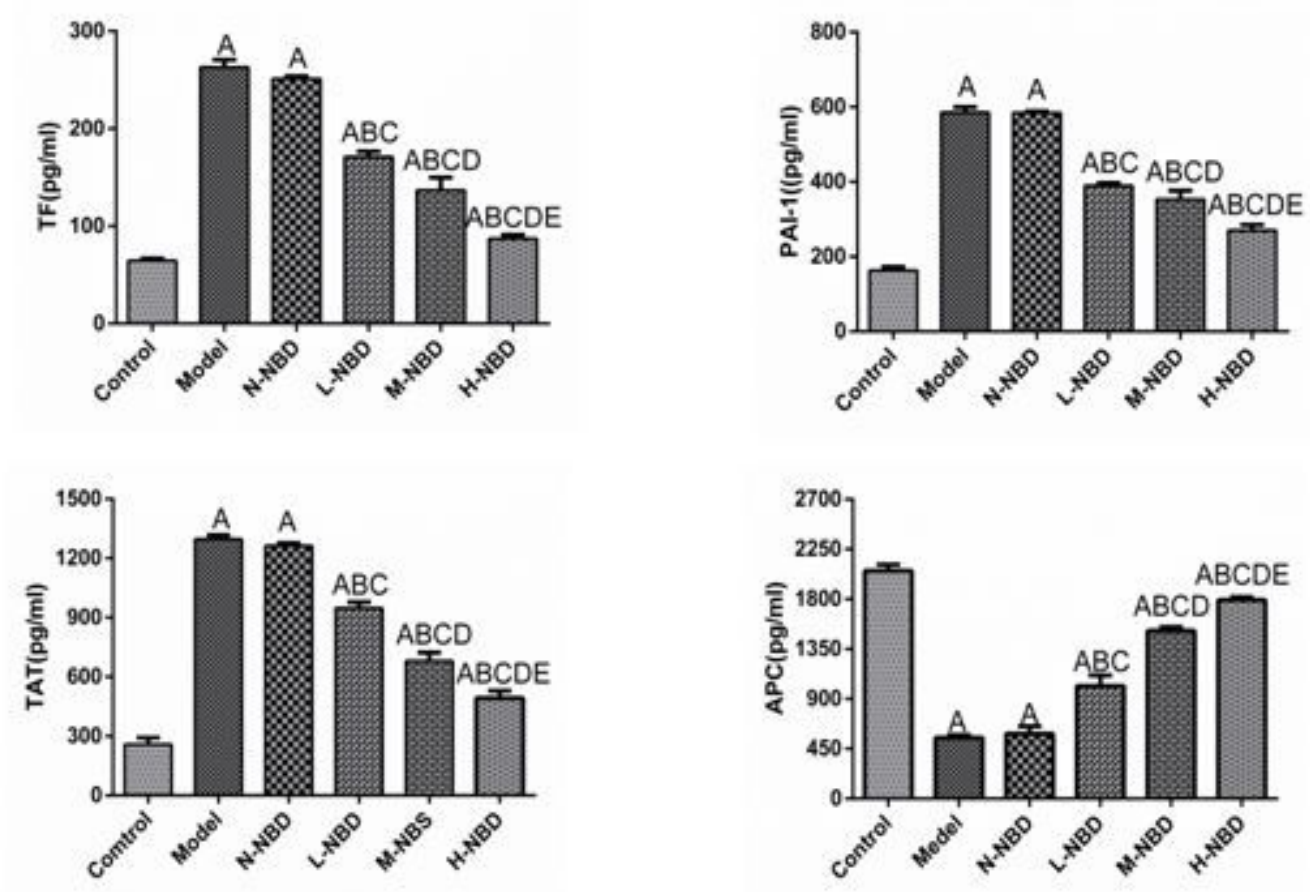

Figure 4

NBDP significantly inhibited TF, PAI-1, TAT, while promoted APC secretions in LPS-induced lung tissues. ELISA showed the changes of TF, PAI-1, TAT and APC secretions following LPS stimulation with or without NBDP. Values were presented as mean \pm SD. Ap $<0.05$ compared with Control. $B p<0.05$ compared with Model. Cp<0.05 compared with N-NBD. Dp<0.05 compared with L-NBD. Ep<0.05 compared with MNBD. 


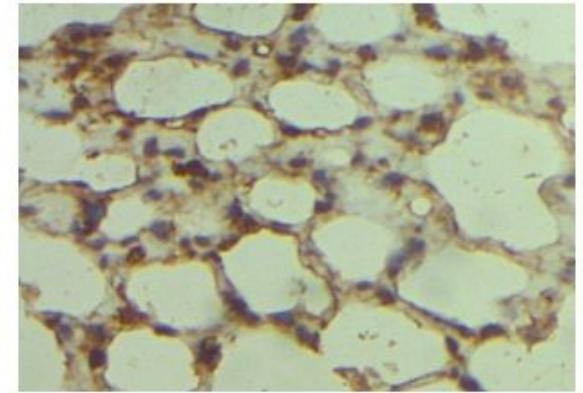

a

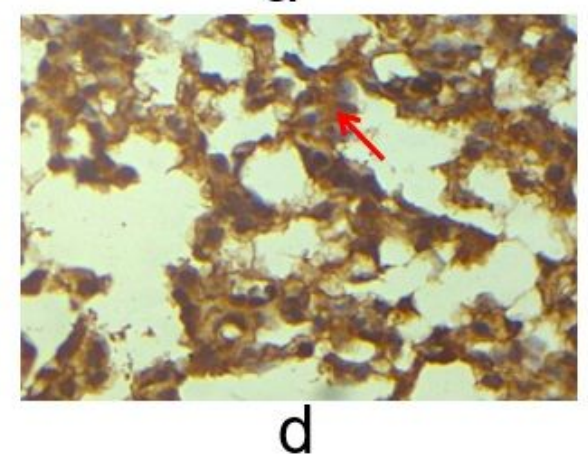

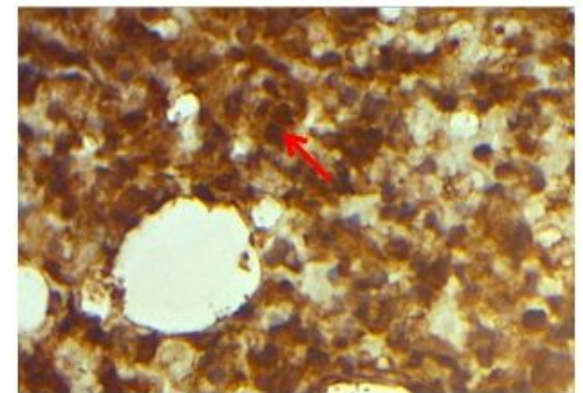

b

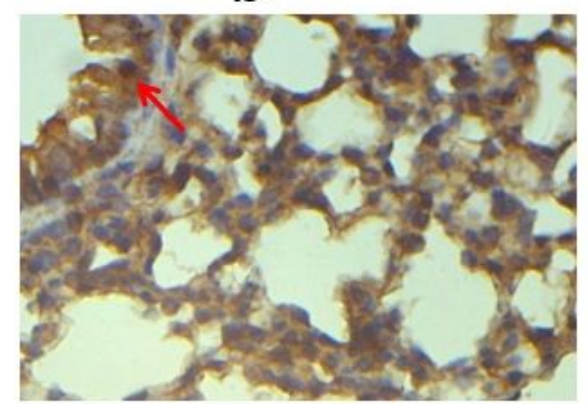

e

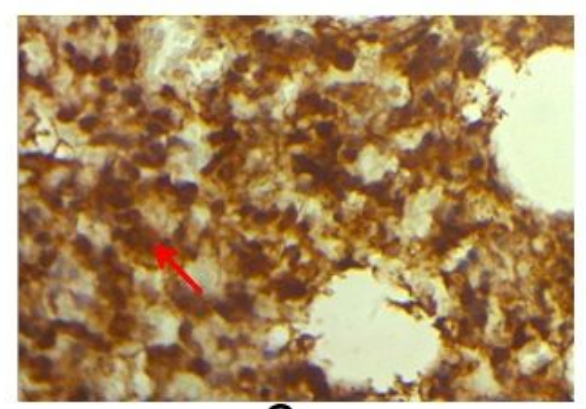

C

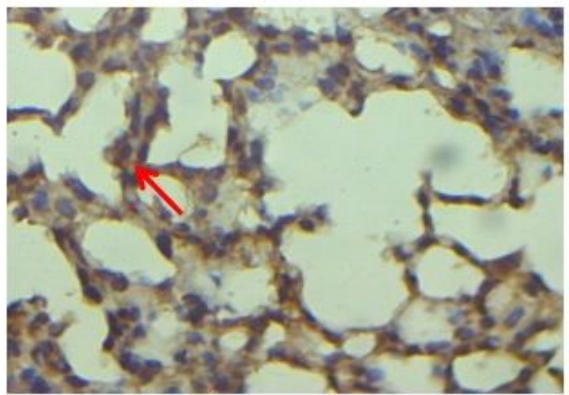

$f$

Immunohistochemical staining $(\times 200)$

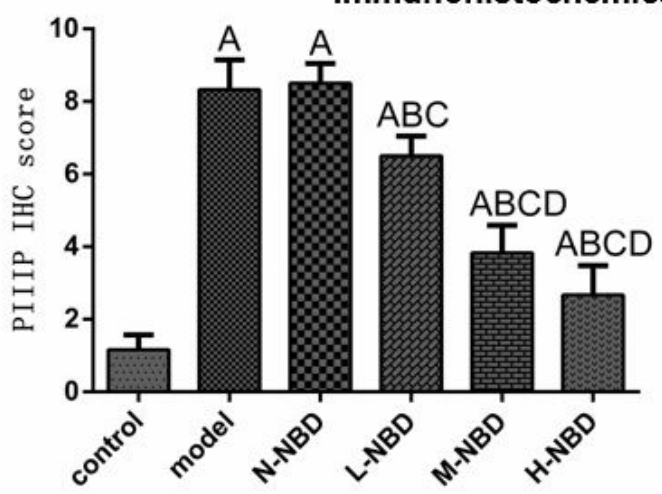

Figure 5

Expression of PIIIP in lung tissues in LPS-induced lung injury mice. PIIIP reflected the level of fibrosis in lung tissues and it was highly expressed in Model and N-NBD group (Brown staining, red arrow). With the NBDP pretreatment, expression of PIIIP significantly decreased. Each bar represents the mean \pm SD. $A p<0.05$ compared with Control. Bp $<0.05$ compared with Model. $C p<0.05$ compared with N-NBD. $D p<0.05$ compared with L-NBD. 
Control Model N-NBD L-NBD M-NBD H-NBD
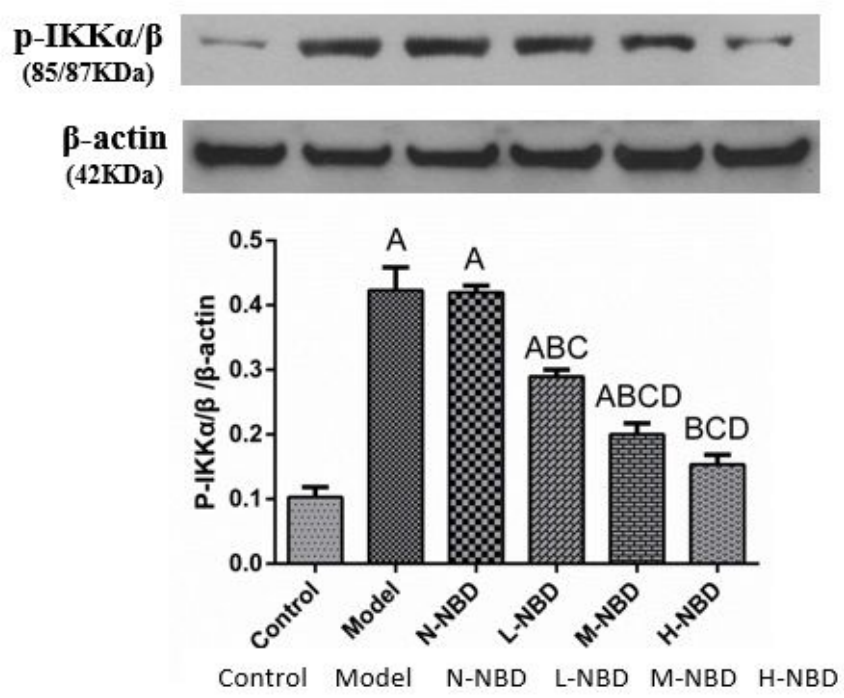

p-IкBa $(40 \mathrm{KDa})$
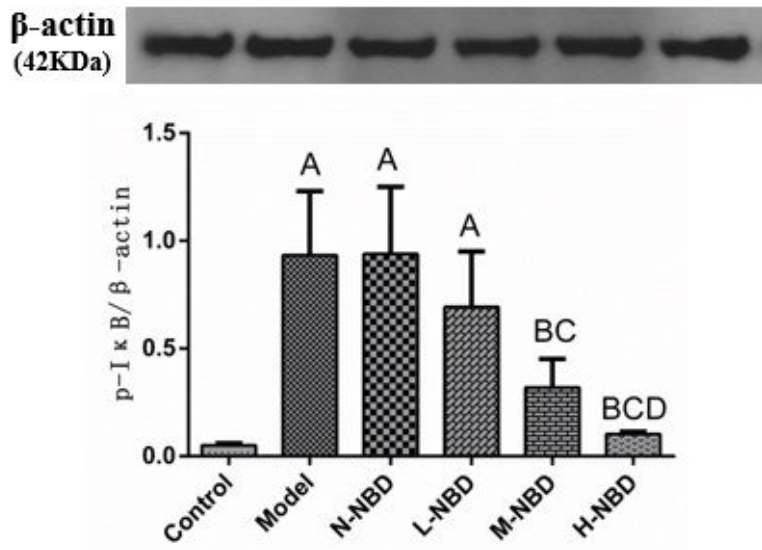

Control Model N-NBD L-NBD M-NBD H-NBD

IkBo

(40KDa)

p-actin

(42KDa)
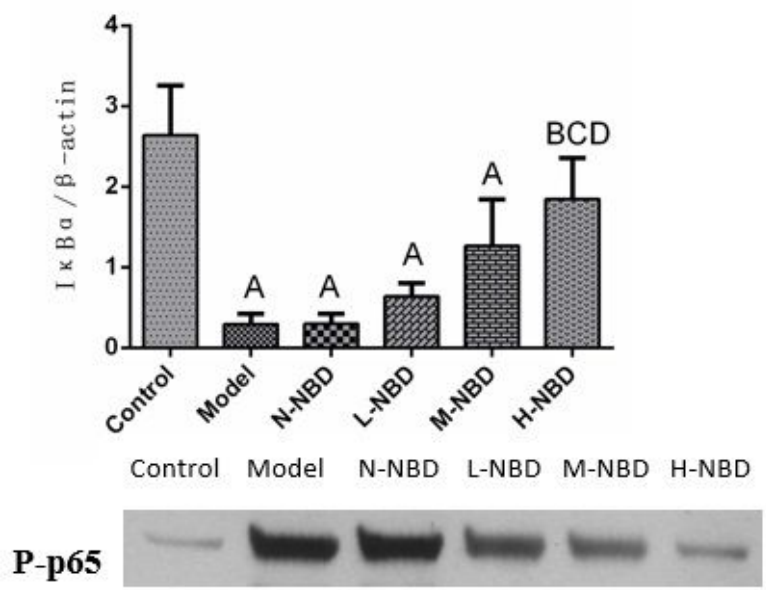

P65 (65KDa)

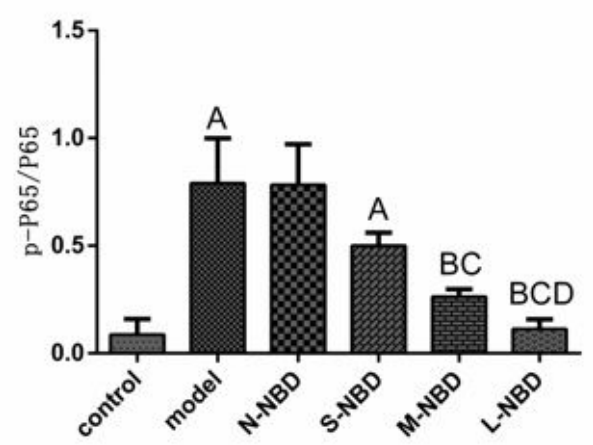

\section{Figure 6}

Expression of PIIIP in lung tissues in LPS-induced lung injury mice. PIIIP reflected the level of fibrosis in lung tissues and it was highly expressed in Model and N-NBD group (Brown staining, red arrow). With the NBDP pretreatment, expression of PIIIP significantly decreased. Each bar represents the mean \pm SD. Ap $<0.05$ compared with Control. Bp $<0.05$ compared with Model. C $p<0.05$ compared with N-NBD. $D p<0.05$ compared with L-NBD. 


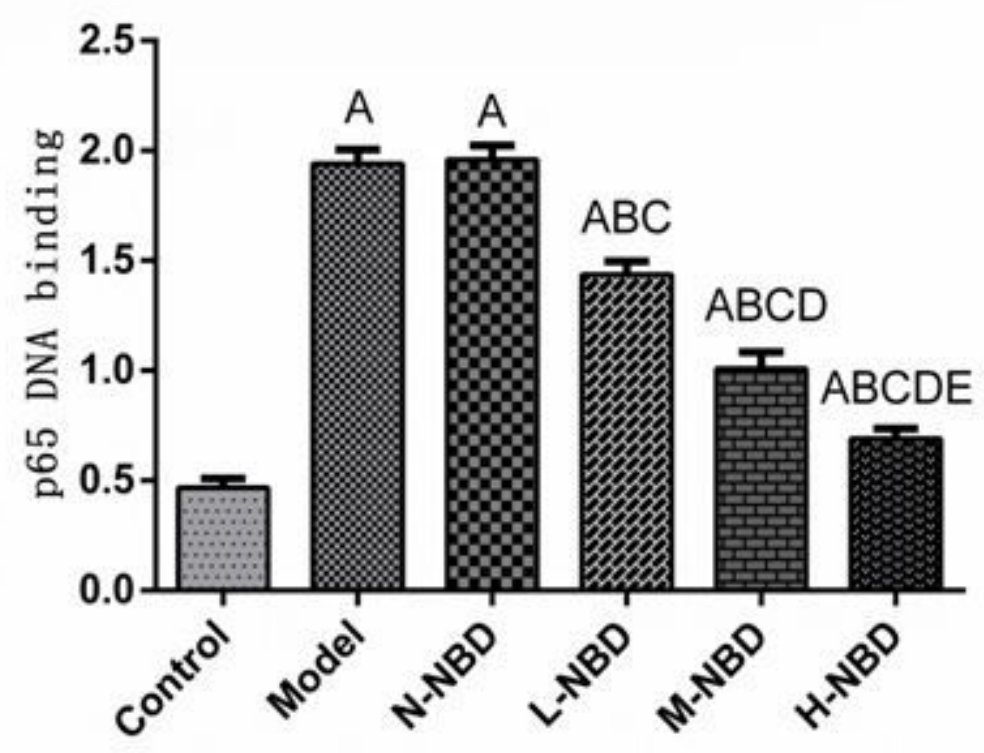

Figure 7

NBDP decreased the inhanced p65 DNA binding activity arisen by LPS inhalation. DNA binding activity of NF-KB p65 was examined by a TransAM p65 transcription factor ELISA kit. Each bar represents the mean \pm SD of 6 mice. Ap $<0.05$ compared with Control. $B p<0.05$ compared with Model. $C p<0.05$ compared with N-NBD. Dp $<0.05$ compared with L-NBD. Ep $<0.05$ compared with M-NBD. 